\title{
Optimizing Image Denoising for Long-Range Brillouin Distributed Fiber Sensing
}

\author{
Marcelo A. Soto ${ }^{(}$, Member, IEEE, OSA, Jaime A. Ramírez, and Luc Thévenaz ${ }^{(}$, Fellow, IEEE, Fellow, OSA
}

\begin{abstract}
Linear and nonlinear 2-D image processing approaches are analyzed with the aim of removing the noise from data acquired by distributed optical fiber sensors based on Brillouin optical time-domain analysis (BOTDA). The impact of the filter parameters on the denoised data is analyzed, especially for the nonlinear image denoising method called non-local means (NLM). In particular, an optimization procedure to find the optimal parameters of the NLM method for BOTDA data denoising is proposed. The described optimization procedure has enabled, to the best of our knowledge, the first experimental demonstration of a conventional BOTDA scheme (i.e., with no modifications in the layout) capable of measuring along a $100-\mathrm{km}$ sensing range over a $200-\mathrm{km}$ fiber loop, using a spatial resolution of $2 \mathrm{~m}$, a frequency sampling step of $1 \mathrm{MHz}$, and reaching a frequency uncertainty of $0.77 \mathrm{MHz}$ with 2'000 averaged time-domain traces. The experimental sensing performance here achieved has been evaluated with a figure of merit (FoM) of 225'000. This is the highest FoM reached without hardware sophistication in BOTDA sensing.
\end{abstract}

Index Terms-Brillouin scattering, distributed optical fiber sensors, image denoising, optical fibers, strain and temperature measurements.

\section{INTRODUCTION}

A FTER almost three decades of research and development, distributed optical fiber sensors based on stimulated Brillouin scattering (SBS) have shown to have relevant and unmatched advantages compared to other distributed sensing techniques, especially when aiming at very long sensing ranges. The time-domain interrogation approach based on a pump-probe SBS interaction, named Brillouin optical time-domain analysis (BOTDA) [1], has allowed for distributed temperature and strain sensing over several tens of kilometers, with spatial resolutions typically of a few meters [2]. Today, the factors ultimately limiting the performance of BOTDA sensors have been clearly identified. On the one hand, the intrinsic loss of the sensing fiber imposes a fundamental limit [2], since it reduces the power of the

Manuscript received July 31, 2017; revised September 4, 2017; accepted September 4, 2017. Date of publication September 14, 2017; date of current version February 24, 2018. This work was supported by the Swiss Commission for Technology and Innovation under Project 18337.2 PFNM-NM. The work of J. A. Ramírez was supported by the CONICYT under Basal Project FB0008. (Corresponding author: Marcelo A. Soto.)

M. A. Soto and L. Thévenaz are with the Institute of Electrical Engineering, EPFL Swiss Federal Institute of Technology, Lausanne CH-1015, Switzerland (e-mail: marcelo.soto@epfl.ch; luc.thevenaz@epfl.ch).

J. A. Ramírez is with the Advanced Center for Electrical and Electronic Engineering, Universidad Técnica Federico Santa María, Valparaíso 1680, Chile (e-mail: jaime.ramirez@usm.cl).

Color versions of one or more of the figures in this paper are available online at http://ieeexplore.ieee.org.

Digital Object Identifier 10.1109/JLT.2017.2750398 optical signals interacting in the SBS process, resulting in low optical power levels reaching the detector. On the other hand, different sources of noise can impact on the BOTDA measurements, imposing serious limitations to the signal-to-noise ratio (SNR) of the measurements [3], [4]. The SNR is actually the parameter ultimately determining the capabilities and performance of BOTDA sensors [2]. Unfortunately, the maximum pump and probe powers that can be launched into the sensing fiber are limited by the onset of nonlinear effects. While the pump pulse power is limited to a critical power of about $100 \mathrm{~mW}$ by modulation instability (MI) [5], the maximum probe power is also constrained to low levels to avoid distortions in the measurements resulting from pump depletion [6], [7].

Several techniques have been proposed in the state-of-the-art to improve the performance of BOTDA systems and to make them more robust against detrimental effects [8]. For instance, using a double-sideband probe scheme, detrimental pump depletion effects can be mitigated [6], while the use of more sophisticated schemes can enable a probe power reaching the ultimate limit imposed by the onset of amplified spontaneous Brillouin scattering [7], [9]. On the other hand, techniques such as optical pulse coding methods [10]-[12], distributed optical amplification (based on Raman or Brillouin scattering) [13]-[15], and time-frequency multiplexing schemes [16]-[18], have allowed increasing the average pump power launched into the fiber, and thus enhancing the measurement SNR while maintaining the peak pump power below the onset of modulation instability. All these sophisticated methods for performance enhancement require some modifications of the conventional BOTDA scheme, which in some cases can lead to complex configurations, mainly when combining several techniques in a single system to reach very long ranges [19], [20]. Recently a technique based on image processing has been proposed [21], [22] to enhance the performance of distributed optical fiber sensors. With no modifications of the classical BOTDA sensor layout, reported experimental results have shown that the SNR can be considerably enhanced by image denoising [21]-[23], obtaining similar or even better performance when compared to other techniques for performance enhancement. Preliminary results have also demonstrated sensing ranges of $100 \mathrm{~km}$ over $200 \mathrm{~km}$ fiber-loop schemes [22], [24]. Although significant SNR enhancements have been obtained in the literature, no descriptions and details have been reported on the optimization procedure of image processing techniques for BOTDA data denoising.

In this paper several approaches for linear and nonlinear image processing methods for BOTDA data denoising are 
described. Special attention is paid to a nonlinear image denoising method called non-local means (NLM), which is optimized to extend the sensing range of conventional BOTDA sensors. The impact of the denoising parameters of this NLM algorithm is described in order to secure a significant SNR enhancement with no loss of information. To the best of our knowledge, this is the first time that an optimization procedure for an image processing method is proposed for BOTDA data denoising. Experimental results validate the proposed optimization procedure, allowing also for the demonstration of the first implementation of a conventional BOTDA sensor scheme (i.e., with no modification on the standard implementation) reaching a sensing range of $100 \mathrm{~km}$, in a $200 \mathrm{~km}$ fiber-loop configuration, with 2 meter spatial resolution and a standard measurement time of a few minutes.

\section{Image Denoising Techniques for BOTDA Data}

The conventional measurement procedure in a distributed Brillouin optical fiber sensor requires the acquisition of the Brillouin gain spectrum (BGS) as a function of distance over the sensing fiber [1], [2]. Acquired data points are commonly stored in a two-dimensional (2D) matrix $\mathbf{g}_{\mathbf{B}}[z, \Delta f]$, in which the value of each position-frequency $[z, \Delta f]$ pair corresponds to the Brillouin gain at a given position $z$ and pump-probe frequency offset $\Delta f$.

In order to reduce noise from the BOTDA measured data, two-dimensional signal processing techniques, such as image denoising algorithms, can be used [21]. The idea exploited in this approach is based on the high level of redundancy of information contained in the data measured by BOTDA sensors [21]-[23]. This means that the $2 \mathrm{D}$ data structure storing the measured Brillouin gain response at each fiber location $z$ for different pump-probe frequency offsets $\Delta f$ contains repeated data patterns, which can be efficiently used to reduce noise. In particular, the Brillouin gain associated to each sampled point in the spatio-frequency domain $[z, \Delta f]$ can be processed by an image denoising procedure mapping the measured SBS gain value into the intensity of a gray-scale (or monochromatic) digital image corrupted by noise [21]-[23].

Image denoising techniques that can be used to reduce noise from BOTDA data are classified as linear and nonlinear [23]. These two approaches are described hereafter. Compared to classical unidimensional filtering methods, image denoising algorithms can be more efficient in terms of noise removal since they can smartly exploit all data patterns existing in the two dimensions of the measured data (this is especially true when using nonlinear image filters).

\section{A. Linear Image Denoising Methods}

Linear image filters are basically two-dimensional linear filters. They use 2D neighbourhood (local) operators to reduce noise from noisy pixels in an image, i.e., from the BOTDA data. The filtering process can be represented by a two-dimensional convolution: $\mathbf{g}_{\mathbf{f}}[z, \Delta f]=\mathbf{h}[z, \Delta f] * \mathbf{g}_{\mathbf{B}}[z, \Delta f]$, where $\mathbf{h}[z$, $\Delta f$ is the spatial impulse response of the filter (spatial kernel in the $z$ and $\Delta f$ domains) [23]. Fig. 1(a) illustrates this 2D

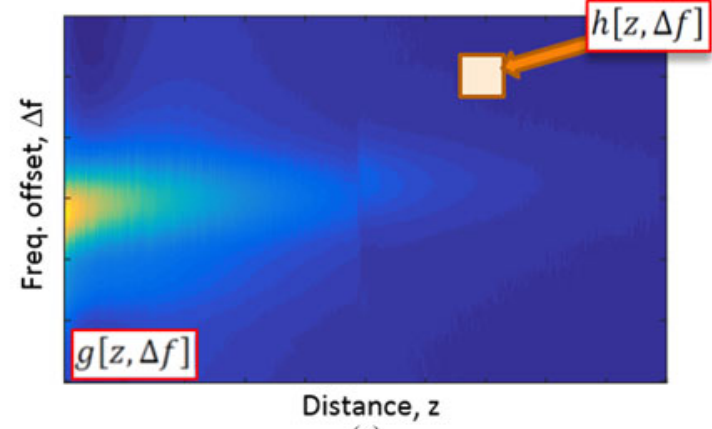

(a)

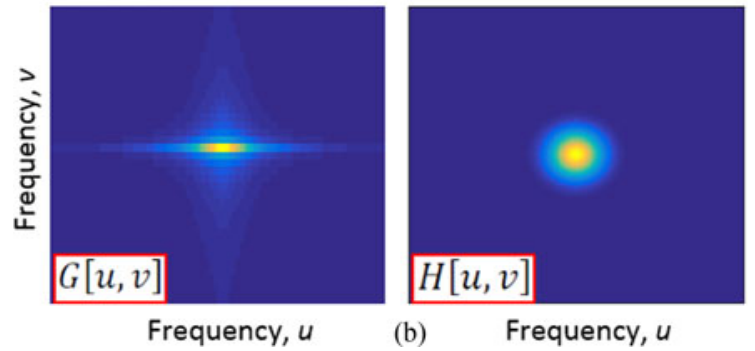

Fig. 1. Approaches for BOTDA data denoising using linear image filters. (a) Approach in the spatial domain, in which the BOTDA data is filtered through a 2D convolution with a filter kernel $\mathbf{h}[z, \Delta f]$. (b) Approach in the frequency domain, in which the 2D Fourier transform of the BOTDA data (left) is multiplied by the 2D Fourier transform of the filter (right).

convolution process, in which the filter $\mathbf{h}[z, \Delta f]$ is used to eliminate noise by processing the BOTDA data directly over the spatial domain $[z, \Delta f]$. Note that, since this kind of filters are linear and space- and time-invariant, this $2 \mathrm{D}$ filtering process can also be represented in frequency domain (see Fig. 1(b)) as the multiplication of the Fourier transforms of the BOTDA data $\mathbf{G}_{\mathbf{B}}[u, v]$ (left) and of the filter spatial response $\mathbf{H}[u, v]$ (right), as: $\mathbf{G}_{\mathbf{f}}[u, v]=\mathbf{H}[u, v] \mathbf{G}_{\mathbf{B}}[u, v]$.

This means that there are two equivalent approaches to perform 2D linear image filtering of the BOTDA data [23], [25]: $i$ ) in the spatial domain (i.e., directly in the $[z, \Delta f]$ space of the BOTDA data) by convolving the data matrix with the 2D filter spatial impulse response, and ii) in the frequency domain by simply multiplying the 2D Fourier transforms of the BOTDA data and filter, and then calculating the 2D inverse Fourier transform of the product. Both approaches are basically equivalent, existing only differences in the processing time of their implementation, which depends basically on the number of data points and on the complexity of the linear filter being used.

One of the main advantages of linear filters is their possible dual description in the frequency and spatial domains of the image. This feature actually makes possible to easily design the spatial and spectral features of the filter. Whilst the proper design of the spatial features is important to secure that the spatial resolution of the BOTDA data is not impaired, spectral features are also relevant to define the amount of noise that is eliminated. Like 1D digital filters, the spectral features of linear image filters are determined by the operating band and bandwidth; however, and unlike 1D filters, here the 2 dimensions of the data must be considered. Interestingly this 2D approach 


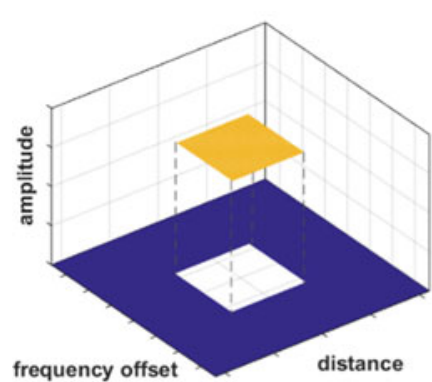

(a)

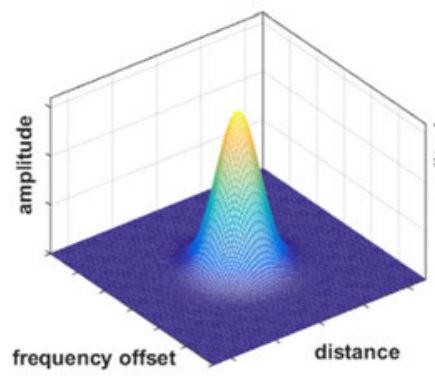

(c)

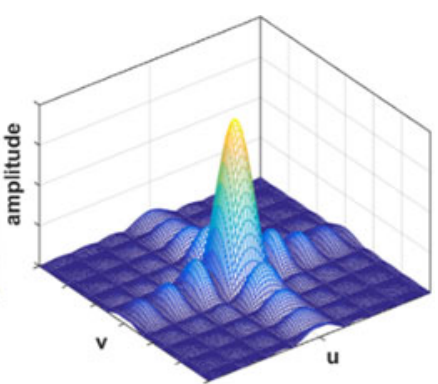

(b)

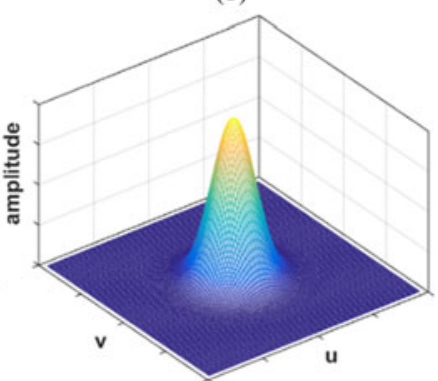

(d)
Fig. 2. Spatial and spectral responses of two linear image filters for BOTDA data denoising. 2D mean filter: (a) Spatial kernel and (b) 2D Fourier transform. 2D Gaussian filter: (c) Spatial kernel and (d) 2D Fourier transform.

allows for the design of the spatial and spectral features of the filters independently over the two dimensions of the data. In the case of BOTDA data, the ratio between the spatial resolution and the sampling interval typically define the size of the spatial kernel $\mathbf{h}[z, \Delta f]$ along the $z$ axis of the data; whilst the BGS width and the scanning frequency step define the kernel size in the $\Delta f$ axis. This implies that not only square spatial kernels could be used, but also rectangular shapes (being longer in the $\Delta f$ axis) can be suitable for BOTDA data denoising.

Note that suitable linear image filters commonly operate as simple 2D low-pass filters, and therefore removing a large amount of noise typically results in blurring and spatial oversmoothing. This feature can lead to a loss of the high-frequency details contained in the BOTDA data, thus reducing the spatial resolving capability of the sensor. However, although having limited denoising capabilities, one of the advantages of 2D linear filters is that they performance does not depend on the data (e.g., on the noise and SNR of the BOTDA measurements). The parameters of the filters can be adjusted based only on the spectral and spatial sampling of the data, and on the original and target signal bandwidths. This feature makes linear filters predictable, offering the possibility of providing a deterministic SNR improvement (for a given original system bandwidth).

Some examples of the most common linear image filters are the $2 D$ mean filter (also known as moving averaging filter) and the 2D Gaussian filter [25]. Fig. 2 shows the spatial and spectral features of these two filters. In particular Fig. 2(a) shows the spatial kernel $\mathbf{h}[z, \Delta f]$ of the $2 \mathrm{D}$ mean filter, which corresponds to a constant value within a well-defined 2D window (this means that all values within this window are averaged with the same weight). This spatial response is actually ideal to constrain the spatial blurring effect within a very well defined 2D spatial window, allowing a good control of the spatial features (in the $[z$, $\Delta f$ ] space) of the denoised BOTDA data (i.e., securing a given well-defined spatial resolution and no impairments on the BGS shape). However, Fig. 2(b) points out that the 2D spectral response of this filter (being a 2D sinc-shaped function) contains spectral sidelobes, implying that high-frequency noise cannot be fully eliminated from the BOTDA data. From this point of view, the $2 \mathrm{D}$ mean filter is not ideal to remove large amount of noise from the data. In order to maximize the noise removal, one might think about the possibility of using a filter with ideal rectangular spectral response, in order to well define the bandwidth of the filter and the amount of removed noise; however such a filter would have a 2D sinc-shaped spatial response, which will deteriorate the spatial information of the sensor. A good trade-off between spatial and spectral responses can be reached with 2D Gaussian filters. Fig. 2(c) and (d) show the spatial and spectral responses of this kind of filter. The curves indicate that while the noise in the BOTDA data can be removed by weighting average within a 2D window centered on each processed data point $[z, \Delta f]$, the $2 \mathrm{D}$ Gaussian filter can more efficiently attenuate high-frequency components beyond a given bandwidth. This way, a good trade-off between noise removal and blurring effect can be achieved with 2D Gaussian filters. For instance, using an isotropic 2D Gaussian kernel with standard deviation of about 2 sampled points, $1 \mathrm{MHz}$ scanning step and sampling interval of $0.5 \mathrm{~m} / \mathrm{pt}$, an SNR improvement of about $7.5 \mathrm{~dB}$ has been reported in a $50 \mathrm{~km}$-long BOTDA sensor with $2 \mathrm{~m}$ spatial resolution [22].

\section{B. Nonlinear Image Denoising Methods}

Note that linear image filters are easy to design and implement; however, they tend to blur sharp edges contained in the data. Thus, when aiming at large levels of noise reduction, lines and other fine image details tend commonly to be lost. To partially overcome this trade-off between spatial smoothing and noise removal, nonlinear image filters can be used. Even though their design and implementation are normally more complex than linear image filters, they can potentially remove much more noise. This feature results in significantly higher SNR enhancement possibilities, while keeping the details of the data. Note that these filters involve the use of a nonlinear function for denoising, which in many cases correspond to time-variant and/or space-variant functions. Therefore, the filtering process cannot be equivalently represented in both space and frequency domain using Fourier analysis, as in the case of linear filters. Based on this feature, there exist two independent categories of nonlinear image denoising methods [23], [25]: i) pixel-wise techniques, in which a nonlinear denoising operator is applied directly to the gain values on the $[z, \Delta f]$ space of the BOTDA data, and ii) transformation-based methods, in which the nonlinear filtering function is applied in a domain representing the frequency content of the data, followed by a process to converted back the denoised data to the original $[z, \Delta f]$ space the BOTDA data. Examples of the first category are the median filter, bilateral filter and non-local means (NLM); whilst transform-based techniques 


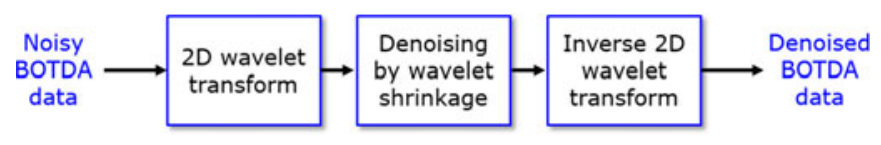

Fig. 3. 2D wavelet denoising approach for BOTDA data.

(second category) can be based, for example, on $2 \mathrm{D}$ discrete cosine transform or $2 D$ discrete wavelet transform [25].

It must be pointed out that the performance of these nonlinear filters and amount of removed noise highly depend on the data itself. Indeed the parameters of the filters typically depend on the features of the BOTDA data, such as on the existing patterns, or standard deviation of the measurement noise [26]-[28]. This implies that the SNR enhancement obtained by nonlinear image denoising cannot be fully predefined by the filter design if the features of the BOTDA measurements are not completely known in advance.

One of the nonlinear image filtering methods that has proved to be efficient for BOTDA data denoising is the $2 D$ wavelet denoising technique [26]. This denoising method requires three steps, as depicted in Fig. 3. In the first one, discrete wavelet transform (DWT) is used to decompose the image defined by the BOTDA data $\mathbf{g}_{\mathbf{B}}[z, \Delta f]$ into multiple frequency bands and small sub-images containing different levels of detail. Using filter banks (low-pass and high-pass filters) and down-sampling, the coefficients obtained by 2D DWT indicate different levels of precision in the decomposition. The second step consists in applying a nonlinear thresholding function (so-called wavelet shrinkage) to the wavelet coefficients obtained by $2 \mathrm{D}$ DWT [26]. In this step, all wavelet coefficients below a given threshold level are associated to noise, and hence set to zero (nonlinear noise elimination function), whilst high-amplitude wavelet coefficients are related to useful information measured by the sensor. Finally, once wavelet thresholding has been applied, inverse discrete wavelet transform is used to reconstruct a denoised version of the BOTDA data in the $[z$, $\Delta f$ ] space using only the remaining high-amplitude wavelet coefficients.

Another example of nonlinear image filtering is the nonlocal means [27]-[29], which takes advantage of the high level of redundancy and similarity contained in the $[z, \Delta f]$ space of the BOTDA data [21]. This approach is indeed ideal for the kind of data measured by BOTDA sensors, which basically consists of a spectral resonance peak that is repeatedly measured at each fiber position. This local resonance peak has in principle the same shape at any fiber location, being only attenuated over distance and spectrally shifted at different fiber positions depending on the temperature and strain distribution along the fiber. This NLM technique searches for 2D data patterns with high level of similarity over the data, being an ideal approach for denoising BOTDA data. More details on this method will be provided in Section III.

Using 2D wavelet denoising and nonlocal means, SNR enhancements of $\sim 14 \mathrm{~dB}$ have been demonstrated in the literature using a $50 \mathrm{~km}$-long classical BOTDA sensor scheme with $2 \mathrm{~m}$ spatial resolution [21].

\section{Designing A NLM Filter For Ultra-Long SEnSing}

Preliminary results have demonstrated the benefits of using the image denoising methods in BOTDA sensing schemes covering ranges over $50 \mathrm{~km}$ [21], [22], whilst much more demanding conditions have also been successfully demonstrated over $200 \mathrm{~km}-$ long loop schemes [22], [24]. Although these relevant experimental demonstrations have been presented in the literature, no descriptions and details on the design of optimal nonlinear image filters have been reported so far.

This work focuses on the optimization of the NLM method for ultra-long BOTDA sensing, aiming at a $100 \mathrm{~km}$ sensing range over a $200 \mathrm{~km}$ fiber-loop scheme. This means that the optimization here is performed under a very much demanding SNR condition when compared to [21], [22], thus testing the ultimate denoising capabilities of the NLM for BOTDA sensing enhancement. Furthermore, a $200 \mathrm{~km}$-long fiber-loop configuration allows for an effective increase in the real remotness of the sensor [16], [19], allowing for the measurement a pont located to a real distance of $100 \mathrm{~km}$ away from the sensor unit. This way the ultra-long sensing capabilities of a standard BOTDA scheme are explored.

It must be noted that in a $200 \mathrm{~km}$ fiber-loop BOTDA system, the probe power is attenuated by $\sim 40 \mathrm{~dB}$ due the intrinsic fiber loss. In order to detect the low-power signal reaching the photoreceiver, a low-noise optical amplifier is usually placed at the receiver front-end as a preamplifier. This makes the BOTDA measurements to be highly dominated by the amplified spontaneous emission (ASE) noise introduced by the amplifier [3]; in particular by the ASE-signal beat noise in the receiver. In the case of a BOTDA sensor, this beat noise can be considered as additive and uncorrelated (white) noise, since the measured time-domain traces are essentially made of a strong DC probe component, topped by a very small Brillouin amplification (about $1 \%$ over the first kilometers of fiber). The Brillouin response has therefore negligible contribution to the total noise of the system [3], and the dominating ASE-signal beat noise can be considered to be independent of the longitudinal evolution of the sensor response, so totally constant and stationary. This condition actually fulfils the requirements for efficient NLM denoising, making the method an effective tool to reduce noise in long-range BOTDA sensors.

In the NLM method, the restored Brillouin gain value attributed to each position-frequency $[z, \Delta f]$ pair is obtained by the weighted average of the gain values of all other pairs in the 2D data structure [21], [27]. In order to calculate the weight associated to each position-frequency pair, neighborhood corresponding to 2D patches and called similarity windows, are defined, as illustrated in Fig. 4. This way, the weighting factors used in the filtering function do not only depend on the pixel values being compared (red dots in the figure), but are calculated to be proportional to the similarity between the entire local neighborhood (squares in the figure) around those data points $\eta(z, \Delta f)$ and the neighborhood of the data point being processed $\eta\left(z_{0}, \Delta f_{0}\right)$. Thus considering that $i=\left[z_{0}, \Delta f_{0}\right]$ is the positionfrequency pair (pixel) being processed and $j=[z, \Delta f]$ represents all other position-frequency pairs in the data $\operatorname{set} \mathbf{g}_{\mathbf{B}}[z, \Delta f]$, 


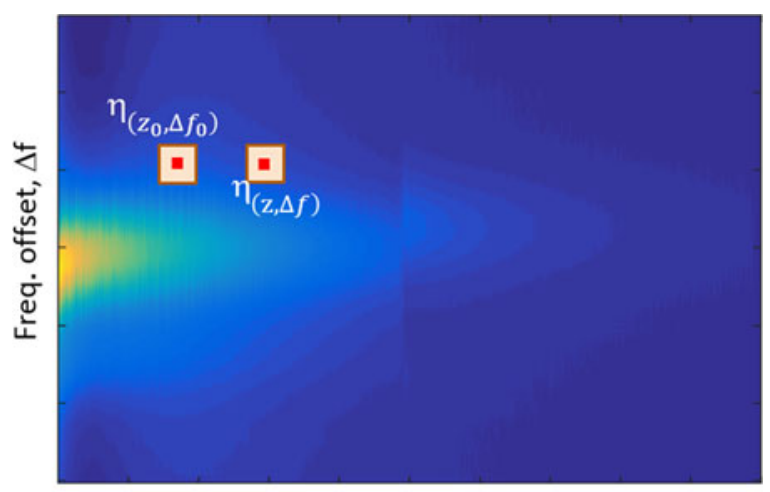

Distance, $z$

Fig. 4. Definition of local neighborhoods (also called similarity windows) in the NLM method. The neighborhood around the data point being denoised $\eta\left(\mathrm{z}_{0}, \Delta f_{0}\right)$ is compared with other neighborhoods $\eta(\mathrm{z}, \Delta f)$ over the entire data set. The red dots represent the pixels (data points) that are being compared in the denoising of $\left[\mathrm{z}_{0}, \Delta f_{0}\right]$, and represent the center pixel of the respective neighborhoods.

the NLM filtering function can be written as [27]:

$$
N L M\left\{\mathbf{g}_{\mathbf{B}}[i]\right\}=\sum_{\forall j} w[i, j] \mathbf{g}_{\mathbf{B}}[j],
$$

where $w[i, j]$ are the weights calculated as the Euclidean distance between the respective neighborhoods divided by the square of a smoothing parameter $h$ [27], so that:

$$
w[i, j]=\frac{1}{k[i]} \exp \left\{\frac{-\left\|g_{B}[i]-g_{B}[j]\right\|^{2}}{h^{2}}\right\},
$$

where $k[i]$ is a normalization factor, which makes the sum of all weights associated to the data point $i=\left[z_{0}, \Delta f_{0}\right]$ equal to 1 .

To avoid loss of information, the size of the similarity window (which is of odd dimensions) has to be smaller than the smallest detail that has to be retrieved in the image. This means that to remove noise in a BOTDA sensor, the similarity window size has to be smaller than the number of digital points sampled within the spatial resolution of the sensor (i.e., smaller than the ratio between spatial resolution and sampling interval). Note that, in the pump-probe frequency offset axis, the typical scanning frequency step of a few $\mathrm{MHz}$ (normally below $5 \mathrm{MHz}$ ) and the BGS spectral width of tens of $\mathrm{MHz}$ generally offer a sufficient spectral sampling, so that limitations on the window size are less restrictive in the $\Delta f$ direction. Based on this approach, it is important to point out that image denoising can only be used in case of oversampling the BOTDA traces in the $z$ axis, while also a sufficient spectral sampling of the BGS is performed. It must however mentioned that the SNR enhancement provided by this NLM method, and also by other nonlinear image denoising methods, is much higher than the SNR improvement obtained by this oversampling factor in linear filtering approaches (typically determined by a square-root dependence on the oversampling factor).

In principle, the search for repeated patterns could be performed by comparing each neighborhood (similarity window) with all other windows existing in the entire 2D data set. However, this can result in very long processing times, especially

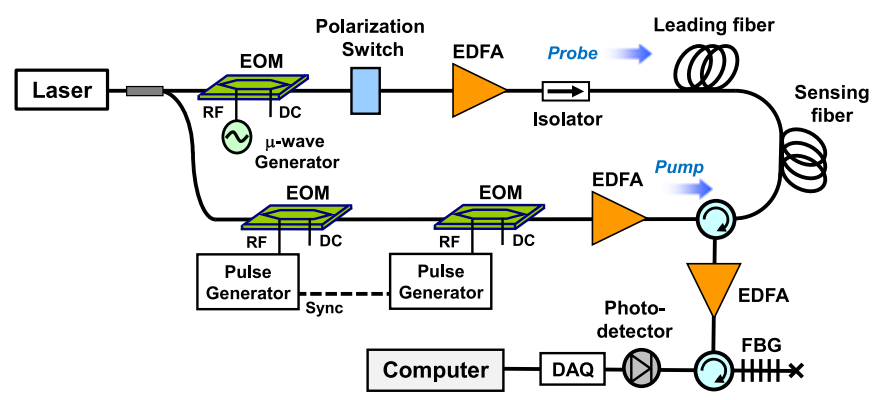

Fig. 5. Experimental setup of a conventional BOTDA sensor scheme using a $200 \mathrm{~km}$ fiber-loop configuration (100 km sensing range).

in a long-range BOTDA sensor in which millions of positionfrequency $[z, \Delta f]$ pairs are typically measured [21]. In order to decrease this computation time, the search for similar neighborhood can be reduced to a searching window [21], [27] centered on the similarity window being processed. The use of this limited searching area also avoids distortions induced by the inclusion of poorly-correlated data into the NLM function described in (1). This makes the NLM denoising to adopt a semi-local approach, which is perfectly compatible with the characteristics of the data measured by a BOTDA sensor (a bell-shaped spectrum with an exponentially decaying behavior over distance), since closely located data points are typically expected to have very close average values [21]. It should be noted that the size of the searching window has to be large enough to secure that several similarity windows are included in the processing and that the algorithm can find enough similar patterns to preserve an optimal level of denoising. However, on the other hand, it should be kept in mind that an extremely large searching window will deteriorate the spatial information and lead to an unacceptably long processing time, which must be dismissed. Thus, an optimal searching window size has to be found for BOTDA sensing data, as it will be described in the analysis of the experimental data presented in Section V-A.

\section{EXPERIMENTAL SETUP}

To optimize the NLM denoising algorithm and evaluate its performance in distributed sensing over ultra-long ranges, a conventional BOTDA scheme [2] has been implemented, with no additional hardware sophistications. Targeting a real remoteness of $100 \mathrm{~km}$ from the sensing unit, and in order to validate the impact of the NLM denoising in very challenging SNR conditions, a $200 \mathrm{~km}$-long fiber-loop configuration is implemented using the experimental setup shown in Fig. 5.

A standard distributed-feedback laser operating at $1550 \mathrm{~nm}$ is here used as optical source. The laser light is split to generate a continuous-wave probe (upper branch in the figure) and a pulsed pump (lower branch). In the lower branch of the setup, a 20 ns pump pulse with high on-off ratio $(>60 \mathrm{~dB})$ is created by cascading two electro-optic modulators (EOMs) of highextinction ratio. Note that, instead of using two cascaded EOMs, pulses with high extinction ratio can also be obtained by using a semiconductor optical amplifier. The pump pulse is boosted by an erbium-doped fiber amplifier (EDFA) up to a peak power of $\sim 100 \mathrm{~mW}$ (limit imposed by modulation instability [5]). The 
sensing fiber is $100 \mathrm{~km}$-long and is composed of two $50 \mathrm{~km}$ spools of standard single-mode fiber having similar Brillouin frequency shift (BFS).

In the upper branch of the setup an EOM of high-extinction ratio $(>40 \mathrm{~dB})$ and driven by a microwave signal is used to generate a two-sideband probe with suppressed carrier [6]. A polarization switch is inserted to mitigate the polarization dependence of the SBS gain. The probe signal is then amplified by an EDFA and launched into a $100 \mathrm{~km}$ leading fiber that is used to convey the probe signal to the farthest end of the sensing fiber (at a real $100 \mathrm{~km}$ distance from the interrogation unit). Note that the use of a leading fiber composed of spools with different BFS has allowed us to increase the input probe power up to $\sim 9 \mathrm{dBm}$, without activating the detrimental amplified spontaneous Brillouin scattering. Considering the losses of the leading fiber $(\sim 21 \mathrm{~dB})$, the actual probe power per sideband entering in the sensing fiber is below $-11 \mathrm{dBm}$, representing a safe probe power to secure that no pump pulse distortions and no nonlocal effects occur over the sensing fiber [6], [7].

In the receiver block, the probe signal containing the Brillouin gain response of the sensing fiber is amplified by a low-noise EDFA. A narrowband $(\sim 7 \mathrm{GHz})$ fiber Bragg grating (FBG) is then used to select the lower-frequency probe sideband and to filter out unwanted spectral components. Note that to increase the reliability of the BOTDA system and minimize performance degradation during operation, an FBG with an athermal packaging is used in this case. The selected probe component is detected by a $125 \mathrm{MHz}$ photodetector and digitalized by a fast data acquisition (DAQ) system connected to a computer.

\section{EXPERIMENTAL RESULTS}

Time-domain BOTDA traces have been acquired with 2'000 averages per scanned frequency (i.e., 1'000 averages for each orthogonal polarization), while 300 frequencies have been scanned with steps of $1 \mathrm{MHz}$. The acquired data points have been arrayed in a $2 \mathrm{D}$ matrix $\mathbf{g}_{\mathbf{B}}[z, \Delta f]$, containing the Brillouin gain response for the different pump-probe frequency offsets $\Delta f$ at each longitudinal point $z$ sampled every $0.5 \mathrm{~m}$ along the fiber. This has led to a matrix $\mathbf{g}_{\mathbf{B}}[z, \Delta f]$ of very large size, containing $200 ’ 000 \times 300$ points.

\section{A. Optimizing the Parameters of a NLM Filter}

Before evaluating the performance of the ultra-long BOTDA sensor here implemented, the parameters of the NLM denoising method are optimized. Note that although the optimal parameters and resulting SNR of the denoised data depend on the features of the measured data itself (e.g., noise standard deviation, spatial resolution, sampling interval, scanning frequency step), the optimization procedure here proposed is valid to denoise data obtained by most of BOTDA sensors, provided that additive Gaussian noise is the dominant kind of noise affecting the measurements.

As described in Section III, there are 3 parameters that must be optimized in the NLM [27]-[29]: the smoothing parameter $h$, the similarity window size, and the searching window size. There exist sophisticated optimization methods developed in the literature based on specific features of natural images [27] [29], however here the features of the BOTDA data are very particular. Indeed some of the denoising approaches to improve the quality of natural images are based on the subjective visual perception; however this is not the right approach to enhance the quality of BOTDA data. Here the proposed optimization procedure is based only on the improvement of an objective metric: the SNR of the data. It must be however mentioned that while the main objective of the optimization is to find the parameters that maximize the SNR, the optimal parameters must also secure no loss of spatial and frequency resolutions.

First, to optimize the size of the similarity window (here assumed to be square, as in the classical implementation of the NLM [27]), it should be considered that the longitudinal sampling interval of the measurements is $0.5 \mathrm{~m}$ and the spatial resolution of the sensor is $2 \mathrm{~m}$. This means that the sensor should be able to detect events as short as 4 longitudinal points (in the $z$ axis), and therefore the similarity window cannot be larger than the number defined by the ratio between spatial resolution and sampling interval. This number of points actually imposes a minimum necessary (but not sufficient) condition to secure no detrimental impact on the spatial resolution due to NLM denoising. Since the similarity window must be of odd dimensions, the only square window size satisfying this necessary condition in this case is $3 \times 3$. This window size secures no data over-smoothing over fiber sections longer than the spatial resolution (4 points). Note also that no impairment on the frequency resolution and BGS spectral shape is expected with this similarity window size, due to the large enough ratio between the BGS spectral width (in this case of $\sim 60 \mathrm{MHz}$ ) and the scanning frequency step of $1 \mathrm{MHz}$. Actually that the similarity window could have been defined of rectangular shape so that a larger number of points could be chosen in the pump-probe frequency offset $\Delta f$ dimension. This sophistication is however not analyzed in this paper, which only focuses on the optimization of the classical NLM algorithm using square windows.

The other two parameters of the NLM algorithm, i.e., the searching window size and smoothing parameter $h$, have both a significant impact on the resulting SNR of the denoised data. It is however important to highlight that both parameters could also have a detrimental impact on the spatial resolution of the sensor. Therefore, the optimization procedure proposed here requires first to measure a hot-spot event of length similar to the target spatial resolution. The optimization of the searching window and smoothing parameter is then performed by an exhaustive experimental analysis by denoising the BOTDA data with a set of different searching window sizes and parameter $h$. The optimal parameters are found to be those ones maximizing the SNR of the denoised data while securing that the amplitude of the hot-spot (i.e., the retrieved temperature shift, or simply the respective BFS change) is not affected (reduced) due to eventual data over-smoothing. For this purpose, a 2 m-long hotspot of $20 \mathrm{~K}$ above the ambient temperature has been induced by heating $2 \mathrm{~m}$ of fiber, near $100 \mathrm{~km}$ distance. Measurements have been continuously repeated in order to have reliable estimations of the noise standard deviation $\sigma$ and of the resulting SNR over consecutive BOTDA measurements. Considering the 


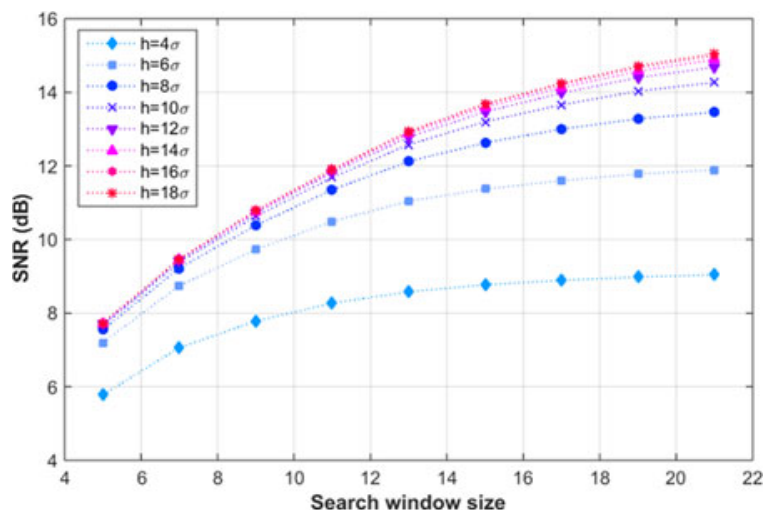

Fig. 6. Impact of the searching window size on the SNR of the denoised data, for different values of smoothing parameter $h$. Searching windows are assumed to be square.

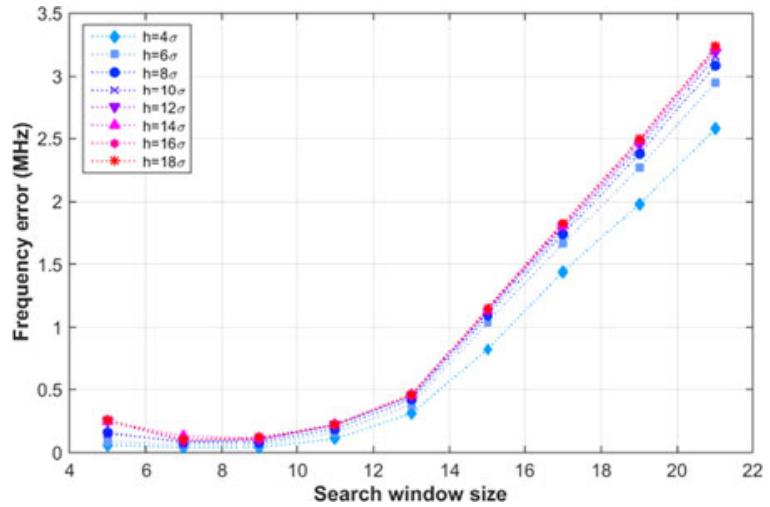

Fig. 7. Error on the retrieved hot-spot amplitude as a function of the searching window size, for different values of smoothing parameter $h$. Searching windows are assumed to be square.

optical pump and probe powers used in the experiment and the number of averages, the noise standard $\sigma$ in this case is equivalent to a Brillouin gain of $0.0114 \%$ at the end of the sensing fiber. This results in an SNR of $\sim 0 \mathrm{~dB}$ at $100 \mathrm{~km}$ distance (SNR over the trace at the peak Brillouin frequency).

Fig. 6 shows the impact of the searching window size on the output SNR for different values of $h$ (ranging from $4 \sigma$ up to $18 \sigma)$. The figure points out that the use of a small searching window size leads to a low SNR enhancement, due to the reduced number of similar patterns that the algorithm can find in a small searching area. Enlarging the searching window actually increases the SNR, but at the expenses of longer processing times. Note that increasing the parameter $h$ also enhances the SNR of the denoised data. However, increasing $h$ beyond 8 or 10 times the noise standard deviation has less impact on the SNR enhancement (this will be further clarified later in the description of Fig. 8). It must be pointed out that the hot-spot measurement plays a key role in the optimization, since it shows the actual spatial capabilities of the sensor. Any over-smoothing of the data as a result of a wrong choice of parameters must be avoided to secure a target spatial resolution. Thus, during the evaluation of different searching window sizes, the effect on the hot-spot amplitude has also been estimated from the retrieved

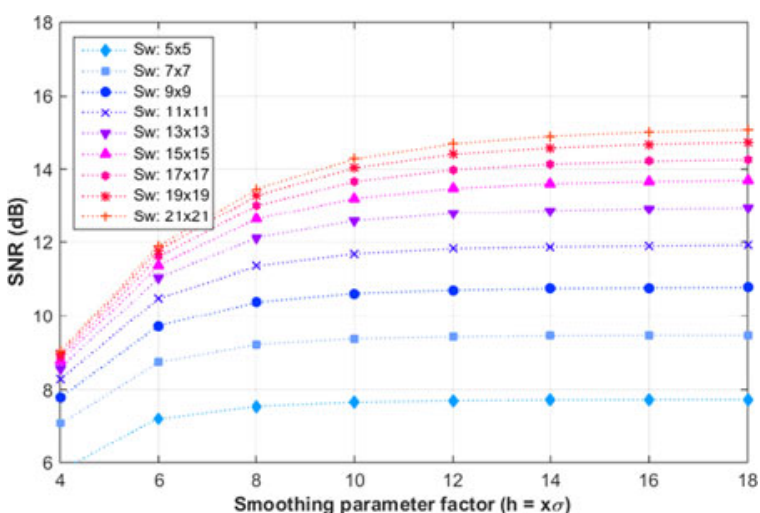

Fig. 8. Impact of the smoothing parameter $h$ on the SNR of the denoised data, for different values of searching window sizes $(\mathrm{Sw})$.

BFS profiles. In order to find the optimal searching window size it becomes important to analyze the impact of this window size on the hot-spot amplitude.

Fig. 7 shows the absolute value of the mean error (over 5 consecutive measurements) in the estimated BFS value inside the hot-spot section with respect to the expected BFS, as a function of the searching window size, for different values of $h$. Results point out that the use of small searching windows leads to a relatively uniform BFS error, determined basically by the remaining noise on the measurements. Those errors are all within the BFS uncertainty of the sensor for windows up to $13 \times 13$. However, when the searching window is increased beyond $13 \times 13$, then the error on the BFS estimation increases significantly as a consequence of an enlarged spatial resolution (i.e., the data is over-smoothed by the NLM image processing algorithm, resulting in a loss of the spatial resolving capabilities of the sensor). Indeed a natural effect of the spatial resolution broadening is the loss of contrast in the retrieved hot-spot value, inducing errors of, for instance, $1.8 \mathrm{MHz}$ and $3.2 \mathrm{MHz}$ for window sizes of $17 \times 17$ and $21 \times 21$, respectively, when using $h=10 \sigma$. These errors could be explained by the low similitude existing between the 2D patches included in the processing for searching windows larger than the optimal size, thus blurring the information contained in the 2D data. In order words, the use of too many uncorrelated, but non-zero, weights in Eq. (1) turns out to blur and deteriorate the spatial features of the information being denoised.

Fig. 8 shows the impact of the smoothing parameter $h$ on the SNR of the denoised data, for the different searching window sizes analyzed in Figs. 6-7. Note that the horizontal axis represents the factor $x$ multiplying the noise standard deviation in the definition of $h=x \sigma$. The figure points out that the use of a high value of $h$ increases the output SNR, especially when using large searching windows. However, when increasing the $h$ beyond $8 \times 10$ times the noise standard deviation only a minor improvement on the SNR is achieved, due to an appreciable asymptotic behavior in the SNR curves. It is however important to notice in Fig. 7 that $h$ has also a small impact on the spatial resolution due to the low-pass filtering effect that introduces. While the error introduced by $h$ is lower than $\sim 0.4 \mathrm{MHz}(\sim 2 \%$ 


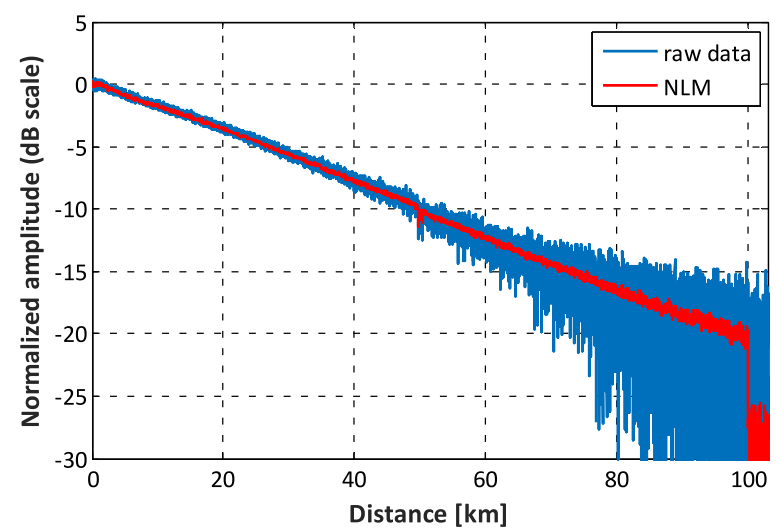

Fig. 9. BOTDA trace at the peak SBS gain frequency, obtained from the raw and denoised data.

of the hot-spot amplitude) for all the analyzed cases (when the window size is below the optimal of $13 \times 13$ ), it still remains important to minimize this error by choosing the lowest possible $h$. It turns out that the use of $h \approx 10 \sigma$ satisfies a condition in which a good enough SNR enhancement is obtained with minor impact on the spatial resolution and hot-spot amplitude (here a tolerance error of $\sim 2 \%$ on the hot-spot amplitude has been accepted). Interestingly, this is exactly the same behavior and conclusion reported in the literature when optimizing the NLM algorithm to denoise natural images [27]. It can therefore be safely inferred that the use of $h \approx 10 \sigma$ is the optimal (or close to optimal) smoothing parameter value for denoising BOTDA data dominated by additive Gaussian noise.

\section{B. Ultra-Long BOTDA Sensing Using Conventional Scheme Over a 200 km-Long Fiber-Loop Configuration}

Using the optimal parameters found in Section V-A (i.e., a similarity window of $3 \times 3$, a searching window of $13 \times 13$ and a smoothing parameter $h \approx 10 \sigma$ ), the actual performance of the implemented long-range BOTDA sensor is evaluated. Note that with the chosen parameters, and using a conventional personal computer with an Intel Core i7-2600 $3.4 \mathrm{GHz}$ processor having 8 cores and a RAM memory of $16 \mathrm{~GB}$, the overall processing time was about 4 minutes in our floating-point Matlab implementation (to denoise the entire matrix of $300 \times 200^{\prime} 000$ data points). Although this time is much shorter than the total measurement time (being $\sim 10$ minutes), this can be highly reduced by a further optimization of the algorithm. For instance, making use of the NLM algorithm implemented in OpenCV library (using an 8 bit representation of the data), the processing time was substantially reduced down to $10 \mathrm{~s}$, indicating the huge potential improvement eventually existing for our Matlab-based implementation.

Fig. 9 shows the raw (blue) and denoised (red) BOTDA traces at the peak Brillouin gain frequency. The figure points out that a large amount of noise is removed from the raw noisy measurements when using the optimized NLM parameters, resulting in a significant increase in the contrast of the BOTDA traces. An SNR improvement of $12.5 \mathrm{~dB}$ is verified in this case. Note that this SNR improvement is lower than the enhancement

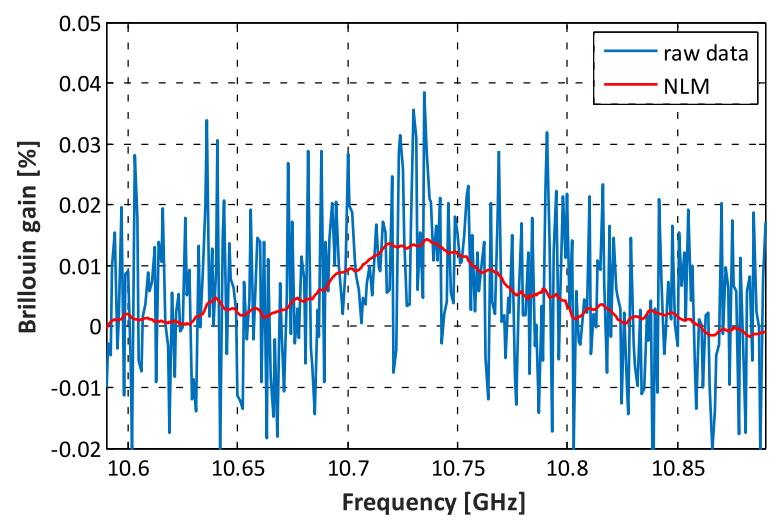

Fig. 10. Brillouin gain spectrum measured near the farthest end of the sensing range (i.e., near $100 \mathrm{~km}$ distance), obtained from the raw and denoised data.

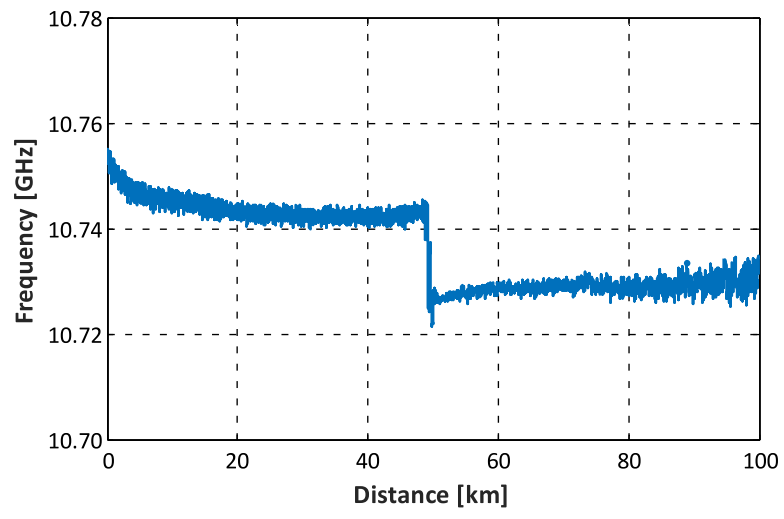

Fig. 11. Brillouin frequency shift profile along the $100 \mathrm{~km}$-long sensing range, obtained from the quadratic fitting over the denoised data.

reported in [21] using similar NLM algorithm. However, it must be pointed out that in this case the SNR of the raw data is practically $0 \mathrm{~dB}$ at the end of the $100 \mathrm{~km}$ sensing distance (after $42 \mathrm{~dB}$ of probe attenuation over the entire $200 \mathrm{~km}$ fiber loop). This condition makes the signal presence extremely tenuous compared to the noise level. Remarkably, the NLM could recover the Brillouin gain response in this case along the entire sensing range, as demonstrated in Fig. 10. This figure actually shows the Brillouin gain spectrum at $100 \mathrm{~km}$ distance for the raw and denoised data. The impact of the optimized NLM image denoising method to recover the gain response contained in the noisy measurement is evident, even under this very low SNR condition. Furthermore no distortions (e.g., broadening and smoothing effects) are observed on the BGS shape obtained after denoising.

The distributed BFS profile over the sensing fiber has been retrieved by using a parabolic fitting [2] of the Brillouin gain spectrum at each fiber location. The estimated BFS profile is shown in Fig. 11. As mentioned before, the $100 \mathrm{~km}$ sensing fiber is composed of two fiber spools having similar BFS; being separated by about $13 \mathrm{MHz}$, as shown in the figure. Note that the thickness of the BFS profile shown in the figure is not representative of the noise and is basically given by the coiling strain in the fibers, inducing BFS oscillations along the entire sensing range. 


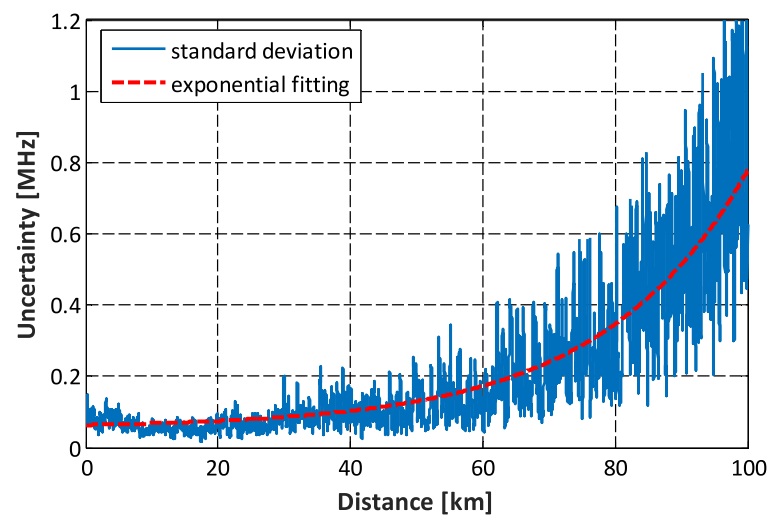

Fig. 12. Frequency uncertainty on the BFS along the $100 \mathrm{~km}$ sensing range, obtained from the denoised data of repeated measurements.

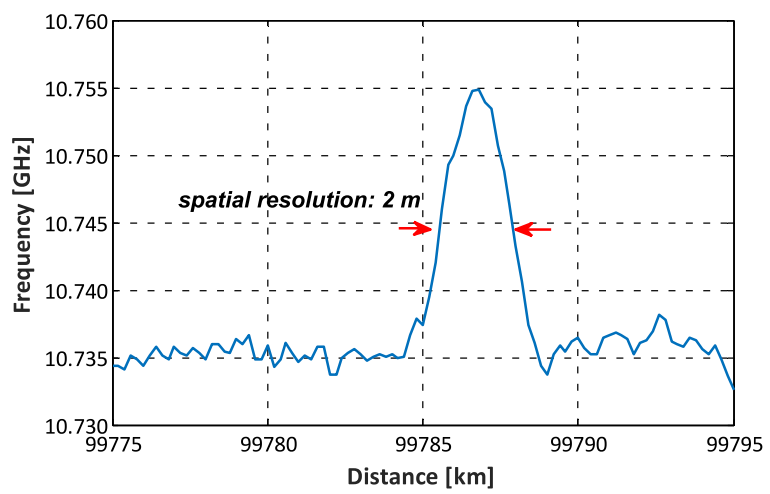

Fig. 13. Demonstration of the $2 \mathrm{~m}$-long hot-spot resolved detection near the sensing range end (i.e., near a $100 \mathrm{~km}$ distance), when using NLM.

To evaluate the uncertainty of the estimated BFS profile, measurements have been continuously repeated, and the standard deviation of the BFS retrieved after denoising has been calculated at each fiber location. The calculated frequency uncertainty versus distance is shown in Fig. 12. The red dashed line corresponds to an exponential fitting of the calculated uncertainty as a function of distance. Results demonstrate that the use of the optimized NLM algorithm leads to BOTDA measurements with a frequency uncertainty of $\sim 0.77 \mathrm{MHz}$ at $100 \mathrm{~km}$ distance.

To verify the spatial resolution of the denoised data, Fig. 13 shows the BFS profile obtained after denoising with optimal NLM parameters around the $2 \mathrm{~m}$-long hot-spot, placed near the far end of the sensing fiber ( $\sim 100 \mathrm{~km}$ distance). The obtained BFS profile demonstrates that the optimized NLM denoising does not distort the data and no loss of information occurs, correctly resolving the $2 \mathrm{~m}$-long spot event.

In order to evaluate the BOTDA performance achieved by this optimized NLM algorithm, the figure-of-merit (FoM) [2] of this implementation has been calculated. Thanks to the large noise removal capability of the NLM method and the use of a $200 \mathrm{~km}$ loop scheme, the achieved performance is rated by a FoM equal to $225^{\prime} 000$. This value corresponds to the highest FoM demonstrated using a standard BOTDA sensor system with no hardware sophistications.
In addition, the denoising capabilities and limits of the NLM method for distributed Brillouin sensing have been further explored by reducing the SNR of the raw measurements below $0 \mathrm{~dB}$. Since only small variations of the SNR have been analyzed, the same optimal parameters of the NLM method found in Section V-A are here used in this analysis. Results have shown that when the SNR decreases below $-3 \mathrm{~dB}$, the NLM algorithm practically cannot recover the data contained in the matrix $\mathbf{g}_{\mathbf{B}}[z, \Delta f]$. In the SNR range between $-3 \mathrm{~dB}$ and $0 \mathrm{~dB}$ the information contained in the BOTDA data can only be partially recovered with very poor repeatability. This behavior can be explained by the fact that when having negative SNR values, the large amount of noise dominates over the real data, preventing image processing from performing a reliable denoising. It can be concluded that, based on this experimental study and on the fundamental principle of the NLM algorithm, an SNR of $0 \mathrm{~dB}$ can be considered to be the lowest SNR in the system for NLM to reliably restore the data contained in BOTDA measurements.

\section{CONCLUSION}

In conclusion, an overview of linear and nonlinear image processing techniques suitable for BOTDA data denoising has been presented. Image denoising has demonstrated to be an efficient and powerful tool to remove noise from measurements of Brillouin distributed optical fiber sensors with no hardware modifications. This feature makes this two-dimensional signal processing approach very attractive for a cost-effective industrial development, since the technique can be readily applied to any existing instrument. The presented analysis points out that whilst the SNR enhancement provided by linear image denoising filters can be deterministically designed based on the spatial and spectral responses of the filter, nonlinear filters offer better denoising capabilities but their performance is more unpredictable, since the provided SNR enhancement highly depends on the data being denoised.

Furthermore, an optimization procedure to find the parameters of a nonlinear image denoising method called nonlocal means has been proposed. Based on the fundamental principle of the method and making use of experimental data, the optimal design of a NLM filter has enabled the implementation of a standard BOTDA sensor scheme covering a sensing range of $100 \mathrm{~km}$ over a $200 \mathrm{~km}$-long fiber-loop configuration, with $2 \mathrm{~m}$ spatial resolution. To the best of our knowledge, this is the first time that a plain BOTDA scheme over such a distance is demonstrated with no hardware modifications, representing the highest FoM $\left(\approx 225^{\prime} 000\right)$ achieved with a standard BOTDA scheme. It is anticipated that image denoising combined with other techniques for BOTDA enhancement, such as optical pulse coding and/or Raman amplification, can lead to a noticeably increased performance, rated by a much larger FoM.

Although the use of image denoising has been here described for long-range BOTDA sensing, the method can be extended (with a suitable adaptation) to other distributed fiber sensing techniques, such as Raman and Rayleigh distributed fiber sensors. This potential extension also includes schemes for 
sub-meter spatial resolution, distributed dynamic sensing or distributed acoustic/vibration sensing, among other schemes.

\section{REFERENCES}

[1] T. Horiguchi, K. Shimizu, T. Kurashima, M. Tateda, and Y. Koyamada, "Development of a distributed sensing technique using Brillouin scattering," J. Lightw. Technol., vol. 13, no. 7, pp. 1296-1302, Jul. 1995.

[2] M. A. Soto and L. Thévenaz, "Modeling and evaluating the performance of Brillouin distributed optical fiber sensors," Opt. Exp., vol. 21, no. 25, pp. 31347-31366, Dec. 2013

[3] M. A. Soto and L. Thévenaz, "Towards 1'000'000 resolved points in a distributed optical fibre sensor," Proc. SPIE, vol. 9157, 2014, Art. no. 9157C3.

[4] J. Urricelqui, M. A. Soto, and L. Thévenaz, "Sources of noise in Brillouin optical time-domain analyzers," Proc. SPIE, vol. 9634, 2015, Art. no. $96342 \mathrm{D}$.

[5] M. Alem, M. A. Soto, and L. Thévenaz, "Analytical model and experimental verification of the critical power for modulation instability in optical fibers," Opt. Exp., vol. 23, no. 23, pp. 29514-29532, Nov. 2015.

[6] L. Thévenaz, S. F. Mafang, and J. Lin, "Effect of pulse depletion in a Brillouin optical time-domain analysis system," Opt. Exp., vol. 21, no. 12, pp. 14017-14035, Jun. 2013

[7] A. Domínguez-López et al., "Novel scanning method for distortion-free BOTDA measurements," Opt. Exp., vol. 24, no. 9, pp 10188-10204, May 2016.

[8] A. Motil, A. Bergman, and M. Tur, "State of the art of Brillouin fiber-optic distributed sensing," Opt. Laser Technol., vol. 78, Part A, pp. 81-103, Apr. 2016.

[9] R. Ruiz-Lombera, J. Urricelqui, M. Sagues, J. Mirapeix, J. M. LópezHiguera, and A. Loayssa, "Overcoming nonlocal effects and Brillouin threshold limitations in Brillouin optical time-domain sensors," IEEE Photon. J., vol. 7, no. 6, Dec. 2015, Art. no. 6803609.

[10] M. A. Soto, G. Bolognini, F. Di Pasquale, and L. Thévenaz, "Simplexcoded BOTDA fiber sensor with $1 \mathrm{~m}$ spatial resolution over a $50 \mathrm{~km}$ range," Opt. Lett., vol. 35, no. 2, pp. 259-261, Jan. 2010.

[11] M. A. Soto, S. Le Floch, and L. Thévenaz, "Bipolar optical pulse coding for performance enhancement in BOTDA sensors," Opt. Exp., vol. 21, no. 14, pp. 16390-16397, Jul. 2013.

[12] S. L. Floch, F. Sauser, M. Llera, and E. Rochat, "Novel Brillouin optical time-domain analyzer for extreme sensing range using high-power flat frequency-coded pump pulses," J. Lightw. Technol., vol. 33, no. 12, pp. 2623-2627, Jun. 2015.

[13] F. Rodriguez-Barrios et al., "Distributed Brillouin fiber sensor assisted by first-order raman amplification," J. Lightw. Technol., vol. 28, no. 15, pp. 2162-2172, Aug. 2010

[14] S. Martin-Lopez et al., "Brillouin optical time-domain analysis assisted by second-order raman amplification," Opt. Exp., vol. 18, no. 18, pp. 18769 18778, Aug. 2010.

[15] J. Urricelqui, M. Sagues, and A. Loayssa, "Brillouin optical time-domain analysis sensor assisted by Brillouin distributed amplification of pump pulses," Opt. Exp., vol. 23, no. 23, pp. 30448-30458, Nov. 2015.

[16] Y. Dong, L. Chen, and X. Bao, "Extending the sensing range of Brillouin optical time-domain analysis combining frequency-division multiplexing and in-line EDFAs," J. Lightw. Technol., vol. 30, no. 8, pp. 1161-1167, Apr. 2012.

[17] Y. Dong, L. Chen, and X. Bao, "Time-division multiplexing-based BOTDA over $100 \mathrm{~km}$ sensing length," Opt. Lett., vol. 36, no. 2, pp. $277-$ 279, Jan. 2011.

[18] M. A. Soto, A. Lavinia Ricchiuti, L. Zhang, D. Barrera, S. Sales, and L. Thévenaz, "Time and frequency pump-probe multiplexing to enhance the signal response of Brillouin optical time-domain analyzers," Opt. Exp., vol. 22 , no. 23, pp. 28584-28595, Nov. 2014.

[19] M. A. Soto et al., "Extending the real remoteness of long-range Brillouin optical time-domain fiber analyzers," J. Lightw. Technol., vol. 32, no. 1, pp. 152-162, Jan. 2014

[20] X. -H. Jia et al., "Hybrid distributed Raman amplification combining random fiber laser based 2nd-order and low-noise LD based 1st-order pumping," Opt. Exp., vol. 21, no. 21, pp. 24611-24619, Oct. 2013.

[21] M. A. Soto, J. A. Ramírez, and L. Thévenaz, "Intensifying the response of distributed optical fibre sensors using 2D and 3D image restoration," Nature Commun., vol. 7, Mar. 2016, Art. no. 10870.

[22] M. A. Soto, J. A. Ramírez, and L. Thévenaz, "Intensifying Brillouin distributed fibre sensors using image processing," Proc. SPIE, vol. 9634, 2015, Art. no. 96342D.
[23] M. A. Soto, J. A. Ramírez, and L. Thévenaz, "Image and video denoising for distributed optical fibre sensors," Proc. SPIE, vol. 10323, 2017, Art. no. $103230 \mathrm{~K}$.

[24] M. A. Soto, J. A. Ramírez, and L. Thévenaz, "200 km fiber-loop conventional Brillouin distributed sensor with $2 \mathrm{~m}$ spatial resolution using image denoising," presented at the Asia Pac. Opt. Sens. Conf., Opt. Soc. Amer., Tech. Dig. 2016, Shanghai China, Paper Th3A.4.

[25] R. Szeliski, Computer Vision: Algorithms and Applications. New York, NY, USA: Springer Science \& Business Media, 2010.

[26] S. G. Mallat, "A theory for multiresolution signal decomposition: The wavelet representation," IEEE Trans. Pattern Anal. Mach. Intell., vol. 11, no. 7, pp. 674-693, Jul. 1989.

[27] A. Buades, B. Coll, and J. M. Morel, "A review of image denoising methods, with a new one," Multiscale Model. Simul., vol. 4, no. 2, pp. 490530, 2005.

[28] V. Duval, J.-F. Aujol, and Y. Gousseau, "On the parameter choice for the non-local means," HAL, Tech. Rep. HAL-00468856, 2010.

[29] J. Salmon, "On two parameters for denoising with non-local means," IEEE Signal Process. Lett., vol 17, no. 3, pp. 269-272, Mar. 2010.

Marcelo A. Soto received the M.Sc. degree in electronic engineering from Universidad Técnica Federico Santa María (UTFSM), Valparaíso, Chile, in 2005, and the Ph.D. degree in telecommunications from Scuola Superiore Sant'Anna (SSSA), Pisa, Italy, in 2011.

In 2006, he was a Lecturer with the Department of Electronic Engineering, UTFSM. During 2010-2011, he was a Research Fellow with SSSA, where he worked on distributed optical fiber sensors based on Raman and Brillouin scattering. From November 2011, he is a Postdoctoral Researcher with the Swiss Federal Institute of Technology, Lausanne, Switzerland, where he has been working on high-performance Brillouin and Rayleigh distributed fiber sensing, nonlinear fiber optics, optical signal processing, and optical Nyquist pulse generation. He is the author or coauthor of about 140 scientific publications in international refereed journals and conferences in the fields of optical communications and optical fiber sensing.

Dr. Soto is a Member of the Optical Society of America, and he is in the Board of Reviewers of major international journals in photonics.

Jaime A. Ramírez received the M.Sc. degree in electronic engineering from Universidad Técnica Federico Santa María, Valparaíso, Chile, in 2007.

From 2006 to 2015, he was a Specialist Engineer and Project Manager in the fields of sensors, data mining, and computer vision for health and mining sectors He is currently the Leader of the Applied R\&D Group with the Advanced Center of Electrical and Electronic Engineering, Universidad Técnica Federico Santa María.

He is author or coauthor of several scientific publications and seven patents in the field of image processing applied to industrial processes.

Luc Thévenaz (M'01-SM'12-F' 17) received the M.Sc. and Ph.D. degrees in physics from the University of Geneva, Geneva, Switzerland.

In 1988, he joined the Swiss Federal Institute of Technology of Lausanne, Lausanne, Switzerland, where he currently leads a research group involved in photonics, namely fibre optics and optical sensing. He achieved with his collaborators the first experimental demonstration of optically controlled slow and fast lights in optical fibres, realized at ambient temperature and operating at any wavelength since based on stimulated Brillouin scattering. He also contributed to the development of Brillouin distributed fibre sensing by proposing innovative concepts pushing beyond barriers. During his career, he was with Stanford University, with Korea Advanced Institute of Science and Technology, with Tel Aviv University, with the University of Sydney, and with the Polytechnic University of Valencia. In 2000, he cofounded the company Omnisens that is developing and commercializing advanced photonic instrumentation based on distributed fiber sensing. His research interests include Brillouin-scattering fibre sensors, slow and fast lights, nonlinear fibre optics, and laser spectroscopy in gases.

Dr. Thévenaz is Fellow of the Optical Society. He is an Associate Editor in three major scientific journals. 